ARTÍCULOS ORIGINALES Rev Chil Salud Pública 2015; Vol 19 (1): 30-36

\section{PERCEPCIÓN DE LA GRAVEDAD DEL CUADRO CLÍNICO COMO DETERMINANTE DE AUTOMEDICACIÓN ENTRE ESTUDIANTES UNIVERSITARIOS}

\author{
Perception of the SeVerity of Clinical symptoms AND \\ SELF-MEDICATION AMONG UNIVERSITY STUDENTS
}

\section{RESUMEN}

Introducción: La automedicación ha sido una estrategia frecuentemente utilizada como respuesta a una situación de malestar o enfermedad, ba jugado y juega un rol importante como primer eslabón en la atención de la salud, aunque no se la reconoce como tal. Se trazó como objetivo analizar la automedicación desde la perspectiva de los propios actores sociales.

Material y método: Investigación de metodología cualitativa entre estudiantes universitarios de la Facultad de Medicina de la Universidad Nacional del Nordeste de Corrientes, Argentina, durante el ciclo lectivo 2013. Se utilizó el Método Comparativo Constante de Glaser y Strauss. Los datos fueron recolectados a través de entrevistas semiestructuradas, en profundidad. Se procedió a un análisis en espiral, arribándose a la generación de categorias respaldadas por fragmentos textuales de las entrevistas.

Resultados: Los estudiantes universitarios se sienten seguros y capaces de a automedicarse ante cuadros clínicos que les resultan de baja complejidad y los previamente vivenciados por ellos o por familiares y conocidos.

Discusión: El sentirse enfermo es una vivencia inseparable de la personalidad y de la biografía de cada sujeto. La respuesta a esa enfermedad es un saber heredado de la historia epigénica oformativa que ha sido olvidada tras su caída en la inmediatez.

Palabras clave: autocuidado, significación, historia formativa, automedicación, estudiantes

\begin{abstract}
Introduction: Self-medication is a frequently used strategy in response to disconfort or illness. It plays an important role in the initial access to primary care, although this is not recognized. The objective of the present study was to analize self-medication from the perspective of the social actors themselves.
\end{abstract}

Recibido 1 de octubre 2014; aceptado 17 de diciembre 2014

Financiamiento: Trabajo subsidiado por la SGCYT-Universidad Nacional del Nordeste PI-17I068. 
Material and Methods: A qualitative methodology was used among university students from the Faculty of Medicine, Universidad Nacional del Nordeste in Corrientes, Argentina, during 2013. The Constant Comparative Method of Glaser and Strauss was used. The data was collected through in depth semi-structured interviews. Spiral analysis was applied, generating categories supported by textual fragments from the interviews.

Results: We found university students feel safe and able to self-medicate for clinical symptoms of low complexity, and symptoms previously experienced by themselves, or by relatives and acquaintances.

Discusion: Feeling ill is an experience inseparable from the personality and biography of the individual. The answer to illness is an inherited knowledge of the epigenetic or formative history that gets forgotten when it falls into immediacy.

Key words: self-care, significance, formative history, self-medication, students.

\section{INTRODUCCIÓN}

Desde la antigüedad y por mucho tiempo el cuidado del enfermo en los hogares ha sido responsabilidad de las mujeres, lo que se conoce hoy como "cuidado genérico", el cual era realizado gracias a un conjunto de conocimientos y habilidades transmitidas culturalmente.

Posteriormente, cuando se organizaron y desarrollaron las comunidades, surgieron las figuras del curandero, brujo, chamán.

Más tarde, emerge la figura del médico, que llevó a una profesionalización del cuidado de la salud y la imposición de un modelo de salud hegemónico, biomédico, donde la opinión del profesional no debía ser cuestionada y los pacientes solamente debían acatar lo indicado.

La autoatención ${ }^{1}$ puede definirse como las representaciones y prácticas que la población utiliza a nivel del sujeto y grupo social para diagnosticar, explicar, atender, controlar, aliviar, aguantar, curar, solucionar o prevenir los procesos que afectan su salud en términos reales o imaginarios, sin la intervención de curadores profesionales, aun cuando éstos puedan ser referencia de esta actividad. Es un término muy amplio, que abarcaría la higiene general y personal, la nutrición, el estilo de vida, los factores ambientales y socioeconómicos, como también la automedicación.

Uno de los mejores análisis teóricos sobre la autoatención ha sido realizado por el antropólogo argentino Eduardo L. Menéndez, ${ }^{1}$ quien afirma que si bien la respuesta de la sociedad a cualquier padecimiento o enfermedad es un hecho cotidiano y recurrente, también es una estructura necesaria para la producción y reproducción de esa misma sociedad. Las personas se constituyen individual y socialmente, por lo que la autoatención puede interpretarse como hecho social, en un contexto histórico y temporal.

La autoatención implica un proceso continuo de síntesis de un grupo social; es el proceso de selección a partir de diferentes experiencias en las que los factores económicos y sociales tienen un rol importante. Este proceso de síntesis permite que la gente resuelva los problemas más frecuentes y recurrentes de salud e incluye nuevas técnicas, productos y creencias que están integradas al saber existente. Parte de esta síntesis es la inclusión o exclusión de productos farmacéuticos para el tratamiento de problemas sentidos o reales.

La automedicación, que forma parte de la autoatención, es una estrategia frecuente utilizada como respuesta a una situación de malestar o enfermedad. Es un acto voluntario de la persona para consigo misma, quien se convierte en sujeto de sus propias acciones. Es todo aquello que las personas hacen por sí mismas con el propósito de reestablecer y preservar la salud o prevenir y tratar las enfermedades. Diversos autores han reflexionado sobre la dificultad de la conceptualización 
de la automedicación. Etimológicamente la palabra está compuesta por el prefijo "auto" (motu proprio), y el lexema "medicación" (medicinas, fármacos). Así, el prefijo "auto" pretende indicar por propia iniciativa, sin mediar prescripción. No obstante, diversos estudios muestran cómo la decisión está influenciada significativamente por los consejos del personal sanitario..$^{2-3}$

Contribuye con la automedicación el hecho de que cada vez es mayor la oferta de salud, mayor es la percepción de la población de enfermedades existentes y de necesidad de curación de ese "padecimiento", con la consecuente medicalización de la vida, ${ }^{1}$ e indica la influencia de la medicina en prácticamente todos los ámbitos.

El mercado del medicamento también contribuiría con la automedicación. Antiguamente existían muchas enfermedades y pocos medicamentos, los cuales eran preparados artesanalmente por los farmacéuticos. La "explosión farmacológica” de los años posteriores a la Segunda Guerra Mundial, consecuencia del gran avance de la química orgánica, de la biología celular y molecular, de la síntesis y semisíntesis química, de la farmacología molecular y sus ramas, farmacocinética, farmacodinamia y farmacoquímica, la genética y la farmacogenética, el advenimiento de los anticuerpos monoclonales, y últimamente el desarrollo de la terapia génica, ha permitido que en poco tiempo se pase a contar con más de 10 mil unidades químico terapéuticas, para el tratamiento de las enfermedades que afectan al ser humano.

Esta "explosión" de nuevos fármacos ha permitido grandes avances en el tratamiento de patologías que antes conducían rápida e inexorablemente a la muerte o a grave incapacidad. Seguramente el incremento de la esperanza o expectativa de vida de las poblaciones que se da en los tiempos actuales se debe, al menos en parte, al enorme progreso ocurrido en la disponibilidad de medicamentos de gran actividad.

Luego de realizar una exhaustiva búsqueda de trabajos relacionados con la automedicación, se ha podido observar que si bien se han realizado numerosos estudios utilizando metodología cuantitativa, ${ }^{4-8}$ no se han encontrado estudios que aborden el tema desde una perspectiva cualitativa o generadora de teoría, lo cual estaría señalando una vacante de estudio.

Frecuentemente se postula que la automedicación se relaciona directa o indirectamente con falta de educación y se plantea la necesidad de "educar a la población" como posible solución. Si esto fuera así, si la educación evitara la automedicación, ¿por qué se automedican estudiantes universitarios? Como expresa Bustos," “... la enfermedad llama siempre a una interpretación que sobrepasa el cuerpo individual y el estado orgánico". [...] "La medicina se inscribe en un espacio social y el médico deberá también aprender del conocimiento y la experiencia del enfermo".

Tratando de responder a este cuestionamiento, se trazó como objetivo de esta investigación el analizar la automedicación desde la perspectiva de los propios actores sociales, haciendo hincapié en la indagación de la percepción de la gravedad del cuadro clínico como determinante de automedicación entre estudiantes universitarios.

\section{MATERIAL Y MÉTODO}

Se realizó una investigación utilizando metodología cualitativa entre estudiantes que se encontraban cursando Farmacología, asignatura del cuarto semestre de la Carrera de Medicina de la Facultad de Medicina de la Universidad Nacional del Nordeste (UNNE) de Corrientes, Argentina, durante el ciclo lectivo 2013.

La selección de los estudiantes incluidos fue realizada por muestreo intencional. Fueron incluidos aquellos que refirieron haber recurrido a la automedicación en el último año, habiendo definido automedicación como el consumo sin prescripción médica de medicamentos de venta bajo receta.

Se utilizó el Método Comparativo Constante, lógica de obtención de datos para la teoría fundamentada o anclada de Glaser y Straus, ${ }^{10}$ pero adoptándose la modificación del método de registro utilizado por la profesora María Teresa Sirvent. ${ }^{11}$ 
Fueron entrevistados 18 estudiantes, igual cantidad de hombres y mujeres, de 19 a 21 años de edad. El número de casos incluidos no resulta crucial para este método, ya que no se intenta la verificación de una teoría deducida de supuestos lógicos a través del análisis de variables, sino que se intenta la generación de teoría sobre un hecho social.

El procedimiento elegido para la recolección de los datos, fue la entrevista en profundidad, semiestructurada y desarrollada a la manera de una conversación iniciada por una pregunta introductoria como disparador. $\mathrm{La}$ consigna a responder fue: ¿Por qué se automedican?

La entrevista fue asumida como una interacción social, donde el entrevistado es quien da sentido y significado a la realidad, explicando su visión de la misma; y el entrevistador es quien intenta interpretar esa explicación. Todas las entrevistas fueron transcritas íntegramente y volcadas en un registro a tres columnas. La recolección de datos se realizó hasta alcanzar la saturación teórica.

Luego del registro de las entrevistas se procedió a su estudio y análisis intensivo, para identificar los temas recurrentes a partir de los conceptos emergentes, identificándose categorías y propiedades que dieron origen a la teoría emergente. El control de la subjetividad se realizó por triangulación de investigadores.

Se aclaró al entrevistado sobre la total confidencialidad de la entrevista y se le leyó y explicó la Hoja de información y el Formulario del Consentimiento Informado Verbal.

\section{RESULTADOS}

Luego de haber realizado un muestreo intencional que permitió identificar a los estudiantes que habían recurrido a la automedicación en el último año, se procedió a iniciar las entrevistas para la recolección de los datos.

Todas se iniciaron con la pregunta disparadora, ¿por qué se automedican? registrándose respuestas que expresaban "¿por qué no?”; “¿por qué pregunta?; “¿cuál es el drama?”; “¿qué problema hay si me automedico?”.
Los entrevistados daban la sensación de que la automedicación era una conducta naturalizada, aceptada, no cuestionable, por lo que se procedió a indagar sobre qué los llevaba a automedicarse.

Sus respuesta dieron origen a temas emergentes a través de incidentes o expresiones que hacían referencia a un cierto valor o sentido común, algunas de las cuales y a modo ilustrativo se encuentran incluidas en el texto entre comillas.

El análisis de estos temas recurrentes y su posterior abstracción, permitió la generación de las siguientes categorías conceptuales relacionadas con la automedicación:

1. Sentirse conocedores de la patología: Los estudiantes en las entrevistas dieron la sensación de que el sentirse conocedores de cuál era su patología los autorizaba a tomar una decisión terapéutica sin consulta previa. A tal efecto, la mayoría se expresó diciendo: "me automedico cuando ya sé lo que tengo" o "cualquiera sabe qué hacer”. Al preguntárseles sobre cómo ellos sabían qué tenían, refirieron: "una gripe sabe cualquiera lo que es"; "un dolor de cabezas, ¿quién no sabe?” o “¿quién no sabe qué tiene que tomar si está con dolor de cabeza?". Otros contestaron: "uno ya sabe qué tiene que tomar"; "automedicarse es una pavada”; "¿qué me puede pasar si me medico solo?”. En el registro quedó asentado que todos daban siempre la impresión de que estaban muy seguros de recurrir a la automedicación y que no demostraban temor a tomar una decisión equivocada que pudiera poner en peligro su salud, contestaban sin titubear, con gran seguridad, mirando perplejos, como no entendiendo por qué se les preguntaba sobre una realidad que para ellos era tan natural.

2. El haber padecido un cuadro clínico similar: El haber vivenciado ellos mismos ese cuadro clínico o que lo haya padecido un familiar o un conocido, les genera seguridad, sensación de saber, de autosuficiencia, de poder resolver los casos por analogía. Cuando se les interrogó sobre 
por qué igual no consultaban a un profesional contestaron: "¿para qué voy a ir al médico?"; "cualquiera sabe que hacer"; "nadie consulta por un dolor de cabeza"; "los médicos siempre te dan lo mismo"; "al final te recetan lo que vos hubieras tomado sin consultar". Surgió del interrogatorio que saben qué hacer porque "siempre se hace lo mismo"; "desde que soy chiquita me dan ibuprofeno si me duele la garganta"; "hasta mi abuela sabe qué hacer si tenés fiebre".

3. No se automedicarían ante situaciones clínicas desconocidas o graves. Cuando en las entrevistas surgió el tema de su accionar ante situaciones clínicas de mayor gravedad o cuando se consideraban muy enfermos o no estaban seguros de la naturaleza del cuadro clínico padecido, todos refirieron que en esos casos no se automedicarían y no dudarían en consultar a un médico y se refirieron al hecho expresando que "más vale que si es grave consulto"; "si es grave... si no sé... voy al médico más vale"; "si sé que no entiendo, más vale que me voy al médico"; "si no sé lo que tengo, ahí sí, voy y consulto”. Al preguntárseles por qué en esos casos no se automedicarían expresaron: "porque ahí ya no sé qué hacer"; "si tengo algo que nunca tuve, ya no me voy medicar solo, voy al médico". Quedó registrado que daban la sensación de que en esas circunstancias eran concientes de sus limitaciones y capaces de actuar con racionalidad.

\section{DISCUSIÓN}

Podemos teorizar que la gravedad del cuadro clínico condiciona la automedicación en la población estudiada. El sentirse enfermo es una vivencia inseparable de la personalidad y de la biografía; por eso cada enfermo vive su padecimiento de manera personal y única.

Para la sociología, a diferencia de la visión individualista, el hecho de estar enfermo o sano no es una circunstancia estrictamente individual, sino que tiene mucho que ver con el marco cultural y social del paciente. Considera que el enfermar es una conducta socialmente aprendida y dependiente en gran parte de factores sociales, culturales y psicológicos. ${ }^{12}$

La salud o la enfermedad son realidades construidas por un individuo social, es decir, que revelan la realidad social en la cual son construidas, y esa realidad social es construida de acuerdo a las necesidades de las clases dominantes. ${ }^{13}$

Estas representaciones o modelos que organizan nuestra experiencia, no surgen espontánea ni arbitrariamente sino que, como el hombre es un ser bio-psico-social, son heredadas de una larga historia formativa o epigenética, biológica, comunal, estatal y societal, donde cada nivel es suprimido, conservado y superado por el siguiente, existiendo entre las partes y el todo un nexo o ligadura funcional. Esa historia resulta olvidada tras la recaída en la inmediatez, es decir que, tras instalarse como estructura, borran su historia formativa y tienden a presentarse como algo dado, como una estructura originaria; a eso puede atribuirse que se registraran en las entrevistas expresiones como "cualquiera sabe qué hacer”. De acuerdo con Durkheim, citado por Samaja ${ }^{14}$ lo normal y lo patológico son nociones relativas a cada especie viviente y en ella, a cada fase de su desarrollo epigenético.

Es evidente que la historia formativa de los estudiantes influye en su praxis ante el enfermar, ya que como hemos podido observar, los estudiantes universitarios ante cuadros percibidos como leves o conocidos, que actúan como sus indicadores o modelos, no dudan en recurrir a la automedicación como primer nivel de atención, porque para ellos "siempre se hace lo mismo".

El actor social, en este caso el estudiante universitario, privilegia y utiliza conocimientos aprendidos que le confieren posibilidad de acción y como desde su perspectiva la automedicación soluciona sus problemas de salud, la incorpora en su historia adaptativa, naturalizándola y preguntándose “¿para qué voy a ir al médico?”.

Que la automedicación se convierta en el primer nivel de atención de la salud, es un dato importante a ser tenido en cuenta por profesionales sanitarios, quienes deberían 
promover la toma de decisiones saludables en estos casos. Cada consulta y cada receta debería acompañarse de indicaciones claras sobre utilización de medicamentos.

Como expresa Samaja, ${ }^{14}$

...todo individuo cuando se enfrenta al mundo de sus objetos actuales, lo bace desde un plexo de prejuicios que su proceso formativo ha depositado en sus instrumentos representacionales (especialmente, en la lengua, en la lógica del pensamiento plasmada en escritura, $y$ en el mundo de las realizaciones tecnológicas). [...] la salud o la enfermedad no son, propiamente hablando, bechos del mundo de las cosas reales o naturales, sino hechos del campo de las relaciones sociales: de las valoraciones subjetivas que los actores hacen sobre los bechos, y de las situaciones de poder que ellos encarnan. La salud es un valor y no un ser: expresa preferencias que se construyen en las situaciones sociales, pero no designa cosas. [...] no bay ni ciencia natural de la salud ni ciencia social [...]. Solo bay construcciones discursivas y rotulaciones, y el unico saber crítico posible es el saber que bace posible la deconstrucción de esos discursos y rótulos: el ejercicio de una pesquisa de su genealogía y, eventualmente, de su denuncia, detrás de un retorno a un origen perdido para siempre.

Ante cuadros desconocidos o vivenciados como graves, los estudiantes universitarios refirieron que no recurrirían a la automedicación y todos manifestaron que consultarían a un facultativo. La significación siempre es social y solo compartiendo los significados con los demás es posible comunicarlos. La producción de significado se hace a través del lenguaje, en una dialéctica entre los sujetos y el entorno sociohistórico. Es por ello que, si bien el médico brinda información al paciente, éste la interpreta y organiza de acuerdo a experiencias y prácticas anteriores, propias y ajenas.

Para la ciencia, los medicamentos a través de sus efectos farmacológicos llevan la idea de bienestar. Estos significados son transmitidos y reforzados por múltiples medios, como las revistas científicas, los médicos, los laboratorios, el marketing, los medios de comunicación, el boca a boca, los que también influyen en la población.

La automedicación está difundida y naturalizada en la población estudiada y es posible que contribuya a esto, el hecho de que detrás de cada medicamento existe un aparato de poder cuyo objetivo es lograr el consumo de la mercancía, un tema para futuras investigaciones. Como cualquier otro producto del mercado, los medicamentos constan de marca, nombre comercial o de fantasía, y publicidad. El mensaje que recibe el consumidor es que los medicamentos estarían en el mismo orden de cosas que aquellas que se adquieren y consumen sin riesgo para la vida. Y dentro de la lógica consumista, cada vez más la salud es entendida como un artículo de consumo.

En cuanto a la forma simbólica que reviste el medicamento, se hace alusión a los significados que una sociedad da a tales objetos. Su significado no es natural, es histórico, dado por una sociedad concreta. Entre el conjunto de significados posibles para el medicamento encontramos que se entiende como equivalente a salud, a bienestar, a necesidad, a solución de problemas, o a prevención. Podríamos afirmar que el medicamento no tiene que ver únicamente con lo terapéutico, es también mercancía y símbolo. El fármaco cura, controla, previene, aliena y domina, representa y simboliza, todo a la vez.

Como señalan Castiel y Álvarez-Dardet Díaz, ${ }^{15}$

... los discursos acerca de la salud significan modos de pensar, escribir, hablar acerca de la salud y sus prácticas. Es necesario ubicar los discursos en determinados momentos históricos y conocer las razones por las que se legitiman al acompañar y ajustarse al orden económico, político y social donde son generados, sostenidos y reproducidos. Los discursos acerca de la salud y los riesgos son construcciones contingentes, de carácter normativo, inapelablemente vinculadas a intereses corporativos y económicos. Además, dependen, de manera explícita o no, de definiciones de lo que sea el ser bumano, 
el tipo de sociedad que se busca y de las maneras de conseguirlo.

El conocimiento al que se ha arribado en esta investigación no se considera definitivo, sino que la teoría alcanzada se asume como un punto superior de arranque para investigaciones futuras, siempre sujeta a una nueva problematización o reformulación. Parafraseando a Samaja, ${ }^{16}$

Un conocimiento científico que no se reincorpore como material y medio de nuevas investigaciones, se torna inútil y se vacía en su noción misma. No solo se desactualiza sino que se vuelve asunto de erudición o alimento de insectos y roedores, pero no un becho de la cultura científica. Sin embargo, [...] los conocimientos científicos pueden reingresar al ciclo de la cultura después de largos periodos de marginación y olvido.

\section{REFERENCIAS}

1. Menéndez E. La enfermedad y la curación ¿Qué es medicina tradicional? Alteridades. 1994; 4(7): 71-83.

2. Caamaño F, Figueiras A, Lado E y GestalOtero JJ. La automedicación: concepto y perfil de sus "usuarios". Gaceta Sanitaria. 2004, 14(4): 294-299.

3. Laporte JR, Castel JM. El médico ante la automedicación. Med Clin (Barc). 1992; 99: 414.

4. Drug Utilization Research Group, Latin America. Multicenter study on self-medication and self-prescription in six Latin American countries. Clin Pharm Ther. 1997; 61: 488-93.

5. Cesolari JAM, Garrote NML, Pérez BM y Busnail LI. Automedicación en adolescentes universitarios. Medicina y Sociedad. 1999; 2(22): 103-106.

6. Grela CA, Álvarez Spence SMC, Georgef Horvat EA, Domínguez JD. Automedica- ción: experiencia en estudiantes de $5^{\circ}$ año de la Facultad de Medicina de la Universidad Nacional del Nordeste. Rev Posgrado de la $V I^{a}$ Cátedra de Medicina [en línea]. 2006; 155: 5-8 [consultado noviembre 2008]. Disponible en: http://med.unne.edu.ar/revista/ revista155/2_155.pdf

7. Achor MS. Storti NI, Arce AJ, Wille Bille JM, Ojeda JA, Bogado Z, Lojo J. Automedicación en el ámbito universitario. Rev de Posgrado de la VI Cátedra de Medicina [en línea]. 2010 Ene [consultado 14.11.2008]; (197) Disponible en: http:// www.unne.edu.ar/Web/cyt/com2004/3Medicina/M-061.pdf.

8. Aguado MI, Núñez MI, Dos Santos Antola L, Bregni C. Automedicación en estudiantes de Farmacia de la Universidad Nacional del Nordeste, Argentina. Acta Farm. Bonaerense. $2005 ; 24(2)$ : 271-6.

9. Kottow M, Bustos R. Antropología Médica. Santiago, Chile: Mediterráneo; 2005.

10. Glaser BG, Strauss A. The discovery of Grounded theory strategic for qualitative research. New York: Aldine Publishing company; 1967.

11. Monteverde AC. Métodos cualitativos. Método comparativo constante. Ficha de Cátedra. Argentina: Universidad de Buenos Aires, Facultad de Filosofía y Letras; 1999.

12. Rubio JM. Realidad y diversidad del enfermar humano (sin número).

13. Diaz E. Entre la tecnociencia y el deseo. Buenos Aires: Editorial Biblos; 2007.

14. Samaja J. Epistemología de la salud: reproducción social, subjetividad y transdisciplina. Buenos Aires: Lugar Editorial; 2004.

15. Castiel LD, Álvarez-Dardet Díaz C. La salud persecutoria. Rev Saúde Pública. 2007; 41(3): 461-466.

16. Samaja J. Epistemología y metodología. Elementos para una teoría de la investigación cientifica ( $3^{\mathrm{a}}$ ed.). Buenos Aires: Eudeba; 2005. 